Subscriber access provided by King Abdullah University of Science and Technology Library

\title{
Article
}

\section{Electronic Properties of a 1D Intrinsic/p-Doped Heterojunction in a 2D Transition Metal Dichalcogenide Semiconductor}

Zhibo Song, Thorsten Schultz, Zijing Ding, Bo Lei, Cheng Han, Patrick Amsalem, Tingting Lin, Dongzhi Chi, Swee Liang Wong, Yu Jie Zheng, Ming-Yang Li, LainJong Li, Wei Chen, Norbert Koch, Yu Li Huang, and Andrew Thye Shen Wee

ACS Nano, Just Accepted Manuscript • DOI: 10.1021/acsnano.7b03953 • Publication Date (Web): 28 Jul 2017

Downloaded from http://pubs.acs.org on August 3, 2017

\section{Just Accepted}

"Just Accepted" manuscripts have been peer-reviewed and accepted for publication. They are posted online prior to technical editing, formatting for publication and author proofing. The American Chemical Society provides "Just Accepted" as a free service to the research community to expedite the dissemination of scientific material as soon as possible after acceptance. "Just Accepted" manuscripts appear in full in PDF format accompanied by an HTML abstract. "Just Accepted" manuscripts have been fully peer reviewed, but should not be considered the official version of record. They are accessible to all readers and citable by the Digital Object Identifier (DOI®). "Just Accepted" is an optional service offered to authors. Therefore, the "Just Accepted" Web site may not include all articles that will be published in the journal. After a manuscript is technically edited and formatted, it will be removed from the "Just Accepted" Web site and published as an ASAP article. Note that technical editing may introduce minor changes to the manuscript text and/or graphics which could affect content, and all legal disclaimers and ethical guidelines that apply to the journal pertain. ACS cannot be held responsible for errors or consequences arising from the use of information contained in these "Just Accepted" manuscripts. 


\section{Electronic Properties of a 1D Intrinsic/p-Doped Heterojunction in a 2D Transition Metal Dichalcogenide Semiconductor}

Zhibo Song, ${ }^{\dagger 1,2}$ Thorsten Schultz, ${ }^{\dagger 3}$ Zijing Ding, ${ }^{\dagger 1,4}$ Bo Lei, ${ }^{1}$ Cheng Han, ${ }^{1,4,5}$ Patrick Amsalem, ${ }^{3}$ Tingting Lin, ${ }^{2}$ Dongzhi Chi, ${ }^{2}$ Swee Liang Wong, ${ }^{2}$ Yu Jie Zheng, ${ }^{1}$ Ming-Yang Li, ${ }^{6,7}$ Lain-Jong Li, ${ }^{7}$ Wei Chen, ${ }^{1,5,8}$ Norbert Koch, ${ }^{* 3}$ Yu Li Huang, ${ }^{2,1}$ and Andrew Thye Shen Wee*1,2,8

${ }^{1}$ Department of Physics, National University of Singapore, 2 Science Drive 3, Singapore 117542, Singapore

${ }^{2}$ Institute of Materials Research \& Engineering (IMRE), A*STAR (Agency for Science, Technology and Research), 2 Fusionopolis Way, Innovis, Singapore 138634, Singapore

${ }^{3}$ Humboldt-Universität zu Berlin, Institut für Physik \& IRIS Adlershof, Brook-Taylor Straße 6, 12489 Berlin, Germany

${ }^{4}$ SZU-NUS Collaborative Innovation Center for Optoelectronic Science \& Technology, Key Laboratory of Optoelectronic Devices and Systems of Ministry of Education and Guangdong Province, Shenzhen University, Shenzhen 518060, China

${ }^{5}$ Department of Chemistry, National University of Singapore, 2 Science Drive 3, Singapore 117542, Singapore

${ }^{6}$ Research Center for Applied Sciences, Academia Sinica, Taipei 10617, Taiwan

${ }^{7}$ Physical Sciences and Engineering, King Abdullah University of Science and Technology, Thuwal 23955-6900, Saudi Arabia

${ }^{8}$ Centre for Advanced 2D Materials, National University of Singapore, Block S14, Level 6, 6 Science Drive 2, Singapore 117546, Singapore

${ }^{\dagger}$ These authors contributed equally to this work.

*Email: phyweets@,nus.edu.sg; huangyl@imre.a-star.edu.sg; nkoch@physik.hu-berlin.de 


\begin{abstract}
Two-dimensional (2D) semiconductors offer a convenient platform to study 2D physics, for example, to understand doping in an atomically thin semiconductor. Here, we demonstrate the fabrication and unravel the electronic properties of a lateral doped/intrinsic heterojunction in a single-layer (SL) tungsten diselenide $\left(\mathrm{WSe}_{2}\right)$, a prototype semiconducting transition metal dichalcogenide (TMD), partially covered with a molecular acceptor layer, on a graphite substrate. With combined experiments and theoretical modeling, we reveal the fundamental acceptorinduced $p$-doping mechanism for SL-WSe $\mathrm{S}_{2}$. At the 1D border between the doped and undoped $\mathrm{SL}-\mathrm{WSe}_{2}$ regions we observe band bending and explain it by Thomas-Fermi screening. Using atomically resolved scanning tunneling microscopy and spectroscopy, the screening length is determined to be in the few nm range, and we assess the carrier density of intrinsic SL-WSe $\mathrm{S}_{2}$. These findings are of fundamental and technological importance for understanding and employing surface doping, e.g., in designing lateral organic-TMD heterostructures for future devices.
\end{abstract}

Keywords: organic-TMD heterostructure, charge transfer, lateral intrinsic/p-doped heterojunction, Thomas-Fermi screening, scanning tunneling microscopy/spectroscopy, photoemission spectroscopy 
Recent progress in two-dimensional (2D) electronic materials has brought fascinating prospective applications in next-generation electronic and optoelectronic devices closer to realization. ${ }^{1}$ A remaining key challenge is finding robust means to controllably select the majority charge carrier type and tune the Fermi level position (i.e., doping) in the 2D semiconductor, as required by the particular device type. ${ }^{2}$ Among various chemical and physical methods, ${ }^{3-13}$ the deposition of organic molecular layers on $2 \mathrm{D}$ materials ${ }^{14-16}$ is a promising doping approach to avoid structural damage, thereby retaining the desired optical and electrical properties of the 2D materials, as such organic layers are mainly stabilized through non-covalent interactions. ${ }^{17}$ Previous studies have revealed that the deposition of suitable molecules can be used to modify the energy levels and the charge density of graphene ${ }^{18-21}$ and semiconducting transition metal dichalcogenides (TMDs) ${ }^{14,15}$ through charge transfer (CT). The situation is more complicated when the heterostructure comprises more than two stacked layers. For example, when graphene is covered with a strong molecular acceptor, it can be $p$-doped or $n$-doped depending on the underlying substrate. ${ }^{18}$ Electrostatic interactions (e.g., screening and dipole fields) in multi-layer systems can significantly impact the interfacial properties. ${ }^{22-24}$ The use of organics also leverages the well-developed organic semiconductor technologies for flexible electronic devices. ${ }^{2,25,26}$

Although much work has been done to understand the electronic properties in vertical ${ }^{27-29}$ and lateral $^{30-32}$ 2D-TMD heterojunctions, there is still a lack of studies of the heterojunctions at the atomic scale. ${ }^{33}$ In particular, the lateral doping of a single layer with a formation of a 1D interface between doped and intrinsic regions is not understood. An ultimate goal is to fabricate lateral heterojunctions with atomically sharp 1D interfaces to facilitate carrier or energy transport for superior device performance, for example, by selective deposition of a dopant material onto well-defined regions of a TMD layer. ${ }^{5}$

In this study, we explain how a molecular acceptor layer on top of a single-layer (SL) tungsten diselenide ( $\mathrm{WSe}_{2}$ ) acts as $p$-type dopant, and reveal the electronic structure at a prototypical 1D interface (a line) between intrinsic and p-doped $\mathrm{WSe}_{2}$ (Figure 1a). Using a combination of lowtemperature scanning tunneling microscopy/spectroscopy (STM/STS) and photoemission spectroscopy (PES) in conjunction with theoretical modeling we explore the molecule/2D semiconductor hybrid on graphite as substrate. The fluorinated fullerene $\mathrm{C}_{60} \mathrm{~F}_{48}$ is chosen as the molecular acceptor as its electron affinity $\left(5.2 \mathrm{eV}^{34}\right)$ is higher than the work function (WF) of the 
$\mathrm{SL}_{-} \mathrm{WSe}_{2}(4.6 \mathrm{eV})$, which should enable CT. Indeed, we find that negative charge is transferred to the acceptor, however, it mainly originates from the graphite substrate while the SL-WSe 2 becomes polarized by the CT dipole field. The semiconductor layer becomes $p$-doped, as evidenced by a $0.7 \mathrm{eV}$ shift of the Fermi level towards the valence band. This doping is further corroborated by the changed transconductance of a field effect transistor (FET) with $\mathrm{WSe}_{2}$ as semiconductor, with and without surface-adsorbed $\mathrm{C}_{60} \mathrm{~F}_{48}$.

Because the molecules adsorb in close-packed islands above a critical coverage, $p$-doped (covered with molecules) and intrinsic (bare) $\mathrm{WSe}_{2}$ regions co-exist next to each other. The border between the two forms a line, i.e., a 1D interface between $p$-doped and undoped semiconductor regions (Figure 1a). Using spatially resolved STS, the Thomas-Fermi (TF) screening length (or Debye length) at such a 1D interface is determined to be in the nm range. This knowledge is important when considering the use of lateral $p-n$ junctions of $2 \mathrm{D}$ semiconductors in future electronic devices.

\section{Results}

Figure 1b shows a typical large-scale STM image of the CVD-grown $\mathrm{SL}_{-W S e_{2}}$ on graphite (Methods section). ${ }^{35}$ As revealed by the atomically resolved image in Fig. 1c, the $\mathrm{WSe}_{2}$ surface away from the edge is clean, smooth and uniform. The $\mathrm{WSe}_{2}$ unit cell is highlighted by a black rhombus with a lattice constant of $3.4 \pm 0.1 \AA$; a typical moiré superstructure arising from the lattice mismatch between graphite and $\mathrm{WSe}_{2}$ is highlighted by a blue rhombus. A proposed structural model of the moiré cell is given in the inset in Fig. 1c, corresponding to a $(3 \times 3)$ supercell of $\mathrm{WSe}_{2}$ with a $(4 \times 4)$ supercell of graphite. The bright half supercell is assigned to Se atoms lying on top of the underlying $\mathrm{C}$ atoms; while the dim half is attributed to the Se atoms located at the hollow sites of the graphite substrates. From the $\mathrm{d} I / \mathrm{d} V$ curve (inset in Fig. 1b), the electronic bandgap of the SL-WSe 2 is deduced to be $1.95 \pm 0.05 \mathrm{eV}$, consistent with previously reported values. $^{24,36}$

$\mathrm{C}_{60} \mathrm{~F}_{48}$ molecules were subsequently deposited onto the clean SL-WSe 2 graphite surface. As shown in Fig. 1d, the molecules aggregate to form close-packed ordered islands with 0.5 ML coverage. The $\mathrm{C}_{60} \mathrm{~F}_{48}$ unit cell is highlighted by the black rhombus in the bottom left inset in Fig. 
$1 \mathrm{~d}$, with a lattice constant of $1.22 \pm 0.04 \mathrm{~nm}$. A similar close-packed $\mathrm{C}_{60} \mathrm{~F}_{48}$ phase is also observed for the $\mathrm{C}_{60} \mathrm{~F}_{48}$ assembly on bare graphite surface. ${ }^{37}$

To obtain insight into the electronic properties at the $\mathrm{C}_{60} \mathrm{~F}_{48} / \mathrm{WSe}_{2} /$ graphite heterostructure, STS measurements were carried out. Figure 2a shows a close-packed molecular island (right) with bare $\mathrm{WSe}_{2}$ (left). Fig. $2 \mathrm{~b}\left(8 \times 8 \mathrm{~nm}^{2}\right)$ is an enlarged part of Fig. $2 \mathrm{a}$ near the $\mathrm{C}_{60} \mathrm{~F}_{48}$ island edge (white square). Unlike the uniform $\mathrm{WSe}_{2}$ surface observed far away from the molecular island or before molecular deposition, a contrast variation is visible close to the island (the upper right corner of Fig. 2b) indicating that the electronic properties of the $\mathrm{WSe}_{2}$ layer are modified. A series of STS spectra assembled in Fig. 2c were obtained at the positions denoted by colored triangles in Fig. $2 \mathrm{a}$ and $2 \mathrm{~b}$. The $\mathrm{d} I / \mathrm{d} V$ spectra obviously vary as a function of the distance $(d)$ from the $\mathrm{C}_{60} \mathrm{~F}_{48}$ island edge. As highlighted by the dashed lines in Fig. 2c, the valence band maximum (VBM) and the conduction band minimum (CBM) shift significantly as the tip moves from the pristine $\mathrm{WSe}_{2}(d>5 \mathrm{~nm})$ towards the $\mathrm{C}_{60} \mathrm{~F}_{48}$ island edge $(d=0 \mathrm{~nm})$. As illustrated in Fig. 2d, the VBM shifts upward to the Fermi level from $+0.84 \mathrm{eV}$ to $+0.22 \mathrm{eV}\left(\Delta U_{\mathrm{VBM}}=\right.$ $0.62 \mathrm{eV})$, while the CBM shifts upwards as well from $-1.10 \mathrm{eV}$ to $-1.48 \mathrm{eV}\left(\Delta U_{\mathrm{CBM}}=0.38 \mathrm{eV}\right)$, consequently implying a bandgap decrease of $\Delta E_{\mathrm{g}}=0.24 \mathrm{eV}$.

The data in Fig. 2 thus strongly suggest that the bare $\mathrm{WSe}_{2}$ is intrinsic and it is $p$-doped when covered by $\mathrm{C}_{60} \mathrm{~F}_{48}$, while a region of band bending forms between the two. It might be straightforward to presume that electron transfer from $\mathrm{WSe}_{2}$ to $\mathrm{C}_{60} \mathrm{~F}_{48}$ causes the $p$-doping of the 2D TMD semiconductor. But previous observations for a molecular acceptor adsorbed on graphene have highlighted the importance of the underlying substrate in tuning the electronic structure of a 2D material, ${ }^{18}$ and thus the graphite substrate must be included in our considerations in due course.

To understand the origin of the CT, we calculated the differential charge density (DCD) across the organic-TMD heterostructure including the graphite substrate, as well as those of reference systems. The side-views of the DCD isosurfaces for $\mathrm{C}_{60} \mathrm{~F}_{48}$ on graphite, SL-WSe 2 /graphite, and SL-WSe ${ }_{2}$ alone are plotted in Figure $3 \mathrm{a}, 3 \mathrm{c}$ and $3 \mathrm{e}$, respectively. A $(7 \times 7)$ graphene supercell and a $(3 \sqrt{3} \times 3 \sqrt{3}) \mathrm{WSe}_{2}$ supercell were used (Methods). The adsorption of the $\mathrm{C}_{60} \mathrm{~F}_{48}$ molecule on a double bond in between hexagons was used in the calculations (SI). The DCD plots reveal that the $\mathrm{C}_{60} \mathrm{~F}_{48}$ molecule is negatively charged in all cases, and the electrons predominantly 
accumulate at the lower half of the molecule. In turn, all substrates are positively charged. However, the sandwiched $\mathrm{WSe}_{2}$ single-layer in Fig. 2c remains essentially charge-neutral but becomes polarized, as the top Se layer facing the molecule is hole-accumulated, while the bottom facing graphite is electron-accumulated.

To provide a more quantitative picture, the plane-integrated $\mathrm{DCD}, \Delta \rho(z)$ (red), and the cumulative $\mathrm{CT}, \Delta Q(z)$ (blue) are plotted in Fig. 3b, 3d and 3f; the latter describes how much charge has been transferred from the bottom to the top at position $z$. The total amount of CT $\left(\Delta Q_{\text {total }}\right)$ from graphite to $\mathrm{C}_{60} \mathrm{~F}_{48}$ reaches -0.40 e per molecule in Fig. $3 \mathrm{~b}$, and reduces slightly to $0.38 e$ with the SL-WSe 2 interlayer in Fig. $3 d$. Two pronounced peaks can be observed in Fig. $3 \mathrm{~d}$ : the top one at -0.38 e represents the $\mathrm{CT}$ from $\mathrm{WSe}_{2}$ /graphite to $\mathrm{C}_{60} \mathrm{~F}_{48}$, and the lower one at $0.25 e$ is that from graphite to $\mathrm{C}_{60} \mathrm{~F}_{48} / \mathrm{WSe}_{2}$. The difference of these two peaks, $-0.13 e$, is then the contribution from the $\mathrm{WSe}_{2}$ interlayer, which is only half of that from the graphite substrate. Consequently, the graphite contributes about two thirds of the charge to the molecular acceptor while the SL-WSe 2 contributes only one third, contradicting the straightforward presumption of dominant TMD-molecule CT. When the graphite substrate is removed, the CT from the $\mathrm{WSe}_{2}$ to $\mathrm{C}_{60} \mathrm{~F}_{48}$ is only $-0.29 e$ as seen in Fig. 3f. The underlying graphite substrate thus plays an important role in defining the electronic structure of the heterojunction, as speculated above.

Further calculations suggest that the $\mathrm{C}_{60} \mathrm{~F}_{48} / \mathrm{WSe}_{2} /$ graphite heterojunction has a CT-induced interfacial dipole moment of $2.65 e \AA$, which is much larger than that of $\mathrm{C}_{60} \mathrm{~F}_{48}$ /graphite without the $\mathrm{WSe}_{2}$ interlayer $(1.70 e \AA)$ and that of for $\mathrm{C}_{60} \mathrm{~F}_{48} / \mathrm{WSe}_{2}$ without the graphite substrate (1.31 $e \AA ̊)$ (SI). The dipole field induced by the CT at the heterojunction can significantly impact the electronic properties (e.g., energy level alignment) and is linked with the CT. In fact, the bandgap decrease of the $\mathrm{WSe}_{2}$ interlayer observed by STS spectra (Fig. 2c) is induced by the vertical electric field, known as Stark effect. ${ }^{38,39}$

To substantiate the conclusions from STM/STS and theoretical modeling, and to highlight the effect of coexisting differently intrinsic/doped $\mathrm{WSe}_{2}$ patches in area-averaging experimental techniques, we performed PES measurements to determine changes of WF (Fig. 4a) as well as valence band (Fig. $4 \mathrm{~b}$ and $4 \mathrm{c}$ ) and core level spectra (Fig. $4 \mathrm{~d}$ ) as a function of $\mathrm{C}_{60} \mathrm{~F}_{48}$ coverage. The spectra of the clean graphite substrate are shown as black dotted lines for comparison. The WF of graphite $(4.5 \mathrm{eV})$ increases by less than $0.1 \mathrm{eV}$ when covered with SL-WSe $2(0 \AA)$, 
indicating negligible electrostatic interaction. After the adsorption of $\mathrm{C}_{60} \mathrm{~F}_{48}$, the WF increases significantly (Fig. 4a). The WF change saturates at a nominal coverage $\Theta$ of $8 \AA$, which is identified as $\mathrm{C}_{60} \mathrm{~F}_{48}$ monolayer coverage. The total $\mathrm{WF}$ increase of $1.56 \mathrm{eV}$ with a $\mathrm{C}_{60} \mathrm{~F}_{48}$ monolayer indicates the formation of an interfacial dipole induced by the CT across the heterostructure, consistent with theoretical modeling.

In Fig. 4b, the SL-WSe 2 shows two distinct features in the valence region at $1.5 \mathrm{eV}$ and $3.9 \mathrm{eV}$ binding energy (BE). The VB edge of the pristine $\mathrm{SL}-\mathrm{WSe}_{2}$, namely the lower binding energy onset of the first peak, is at $1.17 \mathrm{eV}$, slightly larger than the value of the VBM determined by STS $(0.84 \mathrm{eV})$. The most probable reason for the difference is that the VBM of SL-WSe 2 is located at the $\mathrm{K}$ point as determined by ARPES, ${ }^{40}$ however, our VB spectra measured at normal emission are corresponding to electronic states around the $\Gamma$ point. The VB edge around the $\Gamma$ point is energetically deeper than that at the $\mathrm{K}$ point. Therefore, it is reasonable that the VB edge measured by PES, corresponding to the region surrounding the $\Gamma$ point, is further away from the Fermi level than the VBM determined by STS.

The intensities of the two distinct peaks reduce with increasing $\mathrm{C}_{60} \mathrm{~F}_{48}$ coverage. Broadening and BE shifts can be better seen in the zoom-in spectra in Fig. 4c after background-subtraction and normalization. The pristine $\mathrm{WSe}_{2}$ feature at $1.5 \mathrm{eV} \mathrm{BE}$ (red) initially shifts $0.1 \mathrm{eV}$ to lower $\mathrm{BE}$ with the adsorption of $\mathrm{C}_{60} \mathrm{~F}_{48}$. Concomitantly, a shoulder emerges at the lower $\mathrm{BE}$ side and becomes dominant as the $\mathrm{C}_{60} \mathrm{~F}_{48}$ coverage increases. At full monolayer coverage $(8 \AA)$ a single peak at $0.8 \mathrm{eV}$ (orange) remains instead of the pristine $\mathrm{WSe}_{2}$ peak. With accurate fitting, we find that a third contribution (green) is present for the partially covered $\mathrm{WSe}_{2}$ (e.g., $6 \AA$ coverage), which is located halfway between the pristine (red) and the $\mathrm{C}_{60} \mathrm{~F}_{48}$-covered (orange) $\mathrm{WSe}_{2}$ peaks, and is about twice as broad as the other two peaks. The spectral weight of this contribution firstly increases with increasing $\mathrm{C}_{60} \mathrm{~F}_{48}$ coverage and decreases again while approaching monolayer coverage. In accordance with the band bending region existing near the $\mathrm{C}_{60} \mathrm{~F}_{48}$ island edge (Fig. 2), we assign this contribution to the transition area between the $\mathrm{C}_{60} \mathrm{~F}_{48}$ covered and uncovered $\mathrm{WSe}_{2}$. Meanwhile, the peak feature (orange) arising from the $\mathrm{C}_{60} \mathrm{~F}_{48}$-covered $\mathrm{WSe}_{2}$ is constant at $0.8 \mathrm{eV} \mathrm{BE}$ from the very beginning (e.g., $4 \AA$ ), indicating an abrupt change for the $p$-doped $\mathrm{WSe}_{2}$ to the maximum, instead of a gradual shift with the increasing coverage. The $0.7 \mathrm{eV}$ shift of the valence levels towards the Fermi level is identical to the BE shift observed in the core levels, e.g., 
W 4f in Fig. 4d and Se 4d in SI, i.e., all electronic levels shift in parallel due to electrostatic reasons (the CT-induced dipole field). This value is consistent with that of $0.62 \mathrm{eV}\left(\Delta U_{\mathrm{VBM}}\right)$ obtained from STS spectra in Fig. 2.

The STM/STS and PES experiments clearly demonstrate that the intrinsic and $p$-doped $\mathrm{WSe}_{2}$ patches coexist next to each other, and the border between them forms a 1D (line) intrinsic/pdoped interface in the semiconducting $\mathrm{WSe}_{2}$ interlayer. The gradual band bending over several nm observed from the spatially resolved STS spectra (Fig. 2d) is due to in-plane electrostatic screening. Using the non-linear Thomas-Fermi theory to interpret dielectric screening in a semiconductor, ${ }^{41}$ the change of the potential as a function of distance is $\Delta U \propto e^{-d / L_{\mathrm{TF}}}$, where $L_{\mathrm{TF}}$ is the TF screening length (SI). Fitting the curve in Fig. $2 \mathrm{~d}$ with this equation (SI), $L_{\mathrm{TF}}$ of $2.3 \pm 0.7 \mathrm{~nm}$ is deduced in the intrinsic $\mathrm{WSe}_{2}$ side to the hole dopant edge. The in-plane screening length in a 2D semiconductor is a key parameter for applications in electronic devices, and a reliably determined value can aid device design.

Finally, an FET was fabricated to evaluate the carrier densities and the transport properties (SI) of SL-WSe 2 layer without and with $\mathrm{C}_{60} \mathrm{~F}_{48}$. Figure 5a shows the optical microscope image of a back-gate $\mathrm{SL}-\mathrm{WSe} \mathrm{F}_{2}$ FET on a highly $p$-doped silicon substrate with $300 \mathrm{~nm} \mathrm{SiO}_{2}$ (Methods). Transfer characteristics of the FET before and after $\mathrm{C}_{60} \mathrm{~F}_{48}$ deposition were investigated by the source-drain current versus gate voltage, namely $I_{\mathrm{sd}}-V_{\mathrm{g}}$ curve, as shown in Fig. 5b. An ambipolar characteristic is revealed by the inserted logarithmic plot, as $I_{\mathrm{sd}}$ increases with both negative and positive voltages. A much faster current increase is observed in the negative region compared to the positive side, suggesting a hole dominated transport behavior of the intrinsic SL-WSe 2 . By linear extrapolation, the threshold voltage $\mathrm{V}_{\text {th }}$ of the as-made FET device is determined to be 51.6 V for hole transport. After the deposition of $20 \mathrm{~nm} \mathrm{C}_{60} \mathrm{~F}_{48}, \mathrm{~V}_{\text {th }}$ for holes shifts to $-36.6 \mathrm{~V}$, indicating an effective hole-doping. This phenomenon is consistent with the above theoretical calculations shown in Fig. $3 \mathrm{e}$ and $3 \mathrm{f}$, where charge transfer from $\mathrm{SL}-\mathrm{WSe}_{2}$ to $\mathrm{C}_{60} \mathrm{~F}_{48}$ is possible without underlying substrate, analogy to the case with an insulator substrate like $300 \mathrm{~nm} \mathrm{SiO}_{2} / \mathrm{Si}$. The carrier (hole) concentration can be estimated by using the equation $N_{\mathrm{h}}=-C_{\mathrm{i}}\left(V_{\mathrm{g}}-V_{\mathrm{th}}\right) / e$, where $C_{i}$ is the gate capacitance per unit area $\left(1.15 \times 10^{-4} \mathrm{Fm}^{-2}\right.$ for the $300 \mathrm{~nm} \mathrm{SiO}_{2}$ layer $)$. The hole concentration at $V_{\mathrm{g}}=-55 \mathrm{~V}$ is derived to be $2.4 \times 10^{11} \mathrm{~cm}^{-2}$ for the as-made SL-WSe 2 , and it increases to $1.2 \times 10^{12} \mathrm{~cm}^{-2}$ after $\mathrm{C}_{60} \mathrm{~F}_{48}$ doping. 
If the dopant density is known, the TF screening length of a semiconductor can also be estimated by $L_{\mathrm{TF}}=\sqrt{\varepsilon k_{\mathrm{B}} T / e^{2} N_{\mathrm{h}}}$, where $\varepsilon=\varepsilon_{\mathrm{r}} \varepsilon_{0}$ is the dielectric constant (SI). Here, we use $\varepsilon_{\mathrm{r}}=7.25$ for $\mathrm{WSe}_{2},{ }^{42} \mathrm{~T}=300 \mathrm{~K}$ and $N_{\mathrm{h}}=2.4 \times 10^{11} \mathrm{~cm}^{-2}$ obtained from the FET data. This yields $L_{\mathrm{TF}}=1.7 \mathrm{~nm}$ for intrinsic $\mathrm{SL}-\mathrm{WSe}_{2}$ on a $\mathrm{SiO}_{2} / \mathrm{Si}$ substrate at room temperature, which is in good agreement with the value determined by STM/STS on a graphite substrate at $77 \mathrm{~K}$. On the other hand, the carrier density can be estimated from the known $L_{\mathrm{TF}}$, as $N_{\mathrm{h}}=k T \varepsilon / e^{2} L_{\mathrm{TF}}^{2}$. By using $L_{\mathrm{TF}}=$ $2.3 \pm 0.7 \mathrm{~nm}$ obtained from STS, we have $N_{\mathrm{h}}=(4 \pm 2) \times 10^{10} \mathrm{~cm}^{-2}$ at $77 \mathrm{~K}$, which would increase by fourfold to reach the range of $10^{11} \mathrm{~cm}^{-2}$ at room temperature, comparable to the value for the intrinsic $\mathrm{SL}-\mathrm{WSe} \mathrm{S}_{2}$ on $\mathrm{SiO}_{2} / \mathrm{Si}$ obtained from the FET. We thus find high reliability of carrier density and screening length values determined by spatially resolved STM/STS and those derived from transport measurements. We suggest that STS can be used in general to estimate the carrier density in $2 \mathrm{D}$ semiconductors with laterally doped structures, through the determination of the TF screening length at a $1 \mathrm{D}$ interface in a lateral heterojunction.

\section{Conclusion}

The electronic properties of a prototypical molecule/TMD heterostructure on a graphite substrate were elucidated by STM/STS and PES measurements combined with first-principles calculations. The semiconducting SL-WSe 2 can be efficiently $p$-doped by an adsorbed $\mathrm{C}_{60} \mathrm{~F}_{48}$ layer, and a lateral intrinsic/ $p$-doped heterojunction is thereby realized in $\mathrm{SL}-\mathrm{WSe}_{2} . \mathrm{C}_{60} \mathrm{~F}_{48}$ acts as an electron acceptor, however, the negative charge originates mainly from the graphite substrate. In fact, the $\mathrm{WSe}_{2}$ monolayer is strongly polarized by the CT dipole field between graphite and the acceptor layer, and it becomes significantly $p$-doped, accompanied by a bandgap narrowing. The 1D doped/undoped interface features a band bending region that was spatially resolved by STM/STS mapping, and identified as a transition feature in surface-averaged PES spectra. The ThomasFermi screening length of intrinsic SL-WSe ${ }_{2}$ on the graphite substrate was determined to be 2.3 $\mathrm{nm}$ at $77 \mathrm{~K}$ by STM/STS, consistent with the value of $1.7 \mathrm{~nm}$ at room temperature derived from transport measurements in an FET. The comprehensive understanding of the doping mechanism in 2D semiconductors described here is of fundamental importance for developing this technique further. Likewise, it is of technological relevance, as 2D TMD materials and their 
heterojunctions (lateral and vertical) have fascinating prospective applications in future flexible and wearable devices.

\section{Methods}

\section{Sample preparations for STM and PES measurements}

The monolayer $\mathrm{WSe}_{2}$ samples were synthesized by chemical vapor deposition (CVD) method using $\mathrm{WO}_{3}$ powders as precursor. More details about the CVD growth can be found in previous reports. ${ }^{35}$ The sample used for STM measurements was directly grown on a graphite substrate to avoid contaminations introduced by chemical transfer. The overall coverage of SL-WSe $\mathrm{S}_{2}$ is $\sim 10 \%$, estimated by performing statistical analysis over the graphite surface. For PES measurements, a homogeneously large-scale $\mathrm{SL}_{-} \mathrm{WSe}_{2}$ film with size over $5 \times 7 \mathrm{~mm}^{2}$ and coverage higher than $80 \%$ was synthesised on sapphire substrates first and then transferred by a wet chemical method to the graphite substrate. All the $\mathrm{WSe}_{2}$ samples were degassed overnight at $300{ }^{\circ} \mathrm{C}-450^{\circ} \mathrm{C}$ with base vacuum better than $10^{-9}$ mbar before ultrahigh vacuum STM and PES measurements. The cleanliness and quality of the $\mathrm{WSe}_{2}$ crystals were confirmed by STM imaging, STS spectra and XPS wide scan spectra (SI).

$\mathrm{C}_{60} \mathrm{~F}_{48}$ (Nano-C, 99.6\%) molecules were deposited from a Knudsen effusion cell with a typical deposition rate of $\sim 0.04 \mathrm{ML} / \mathrm{min}$. Here, one monolayer (ML) refers to one full monolayer $\mathrm{C}_{60} \mathrm{~F}_{48}$ in close-packed configuration. The deposition rate of $\mathrm{C}_{60} \mathrm{~F}_{48}$ was monitored by a quartz-crystal microbalance (QCM) during evaporation, and the overall coverages were further calibrated by taking statistic over large-scale STM images or monitoring the attenuation in intensity of the C 1s peak during PES measurements. During the deposition, the substrates were held at room temperature and the samples were subsequently transferred in-situ to the attached STM chamber or PES analysis chamber for analysis.

\section{Characterization}

LT-STM studies were performed in an Omicron high-resolution STM interfaced to a Nanonis controller at the National University of Singapore. 6, 24, 36 All STM/STS measurements were recorded at $77 \mathrm{~K}$. An electrochemically etched tungsten tips were used and bias voltages were applied to the STM tip. Tip bias voltage was used in the paper for the convenience to compare 
with PES spectra. STM images were recorded by using constant current mode with a tunnelling current $I_{\text {Tip }}$ of about $0.05 \mathrm{nA}$. For $\mathrm{d} I / \mathrm{d} V$ spectra, the tunnelling current was obtained by a lock-in amplifier, with a modulation of $625 \mathrm{~Hz}$ and $40 \mathrm{mV}$.

All the PES measurements in this study were conducted at Humboldt Universität zu Berlin. For UPS, a Scienta He discharge lamp $(\mathrm{h} v=21.21 \mathrm{eV})$ was used. XPS was conducted using a Scienta DAR 400 x-ray source. The $\mathrm{Mg} \mathrm{K}_{\alpha}$ line $(1253.69 \mathrm{eV})$ was used for excitation. The emitted electrons were detected with a Specs Phoibos 100 hemispherical energy analyzer in fixed analyzer transmission mode and with an acceptance angle of $\pm 7^{\circ}$. The sample was biased at $-10 \mathrm{~V}$ when measuring the secondary electron cut-off (SECO) to overcome the work function of the analyzer. A clean gold crystal was used to calibrate the work function of the detector and to determine the energy resolution $(150 \mathrm{meV})$ of the system. The reproducibility of all energy values in this study was within $0.1 \mathrm{eV}$.

\section{Computation}

The first-principles calculations were carried out based on density functional theory (DFT), employing Projector augmented wave pseudopotentials ${ }^{43,44}$ and the Perdew-Burke-Ernzerholf form $^{45}$ of the exchange-correlation functional, as implemented in the Vienna ab initio simulation package code. $^{46}$ The experimental graphite lattice constant of $2.46 \AA$ was used. The SL-WSe 2 on a single-layer graphene was optimized by using the van der Waals density functional method (optB88-vdW). ${ }^{47,48} \mathrm{We}$ employed an energy cutoff of $400 \mathrm{eV}$ for plane waves, and the criterion for total energy convergence was set to $10^{-4} \mathrm{eV}$. All atoms were relaxed during geometry optimization until the magnitude of forces was less than $0.01 \mathrm{eV} / \AA$. For the plots shown in Fig. 3, a $(3 \sqrt{ } 3 \times 3 \sqrt{ } 3) \mathrm{WSe}_{2}$ supercell on a $(7 \times 7)$ graphene supercell (with stress of $\sim 0.3 \%$ ) including a $\mathrm{C}_{60} \mathrm{~F}_{48}$ molecule and at least $17 \AA$ of vacuum was used. The systems without the SL-WSe 2 interlayer and without graphene substrate were also calculated for comparison. Note that the molecular density in these models is only half of the close-packed phase shown in Figs 1 and 2, more calculations had been performed to understand the effect of the molecular density on the total amount of the charge transfer which will be presented in a paper under preparation. ${ }^{38}$ The calculations based on a model including a 3-layer graphite slab are shown in SI.

\section{Device}


A monolayer $\mathrm{WSe}_{2}$ was mechanically exfoliated from bulk $\mathrm{WSe}_{2}$ crystals using a scotch tape and transferred onto degenerately $p$-doped silicon wafers coated with $300 \mathrm{~nm} \mathrm{SiO}_{2}$. The source and drain electrodes were precisely patterned on the flake using conventional e-beam lithography technique, followed by thermal evaporation of $\operatorname{Pd}(20 \mathrm{~nm}) / \mathrm{Au}(50 \mathrm{~nm})$ as the metal contacts. After liftoff the as-made device was wire bonded onto a leaded chip carrier and loaded in a customdesigned high vacuum system $\left(\sim 10^{-8}\right.$ mbar $)$ for in situ electrical measurements. The transfer characterization $\left(I_{\mathrm{sd}}-V_{\mathrm{g}}\right)$ of the FET devices was carried out by an Agilent 2912A source measure unit at room temperature.

\section{Acknowledgements}

A.T.S.W. acknowledges financial support from MOE AcRF Tier 1 grant number R-144-000321-112 and the Graphene Research Centre. Y.L.H. and D.C. acknowledge the A-STAR SERC grant support for the 2D growth project under the 2D pharos program (SERC 1527000012). Work in Berlin was supported by the DFG (SFB951 and AM419/1-1). Calculations were performed on the Graphene Research Centre cluster supported by Prof. Su Ying Quek.

\section{Author Information \\ Corresponding Author \\ *Email: phyweets@nus.edu.sg (A.T.S.W); huangyl@imre.a-star.edu.sg (Y.L.H.); nkoch@physik.hu-berlin.de (N.K.)}

\section{Author Contibutions}

${ }^{\dagger}$ Z.S., T.S. and Z.D. contributed equally to this work.

\section{Additional information}

Supporting Information Available: Adsorption configurations of $\mathrm{C}_{60} \mathrm{~F}_{48}$ molecules; Theoretical calculations on different systems; XPS full spectrum of the clean $\mathrm{WSe}_{2} /$ Graphite substrate; Se $3 \mathrm{~d}$, $\mathrm{C} 1 \mathrm{~s}$ and $\mathrm{F} 1 \mathrm{~s}$ core levels for $\mathrm{C}_{60} \mathrm{~F}_{48} / \mathrm{SL}-\mathrm{WSe} 2 /$ graphite; Derivation of the change of the electrostatic potential in the SL-WSe 2 on Graphite; Deduce the TF screening length by fitting the 
spatial $\mathrm{d} I / \mathrm{d} V$ spectra; The carrier density for $\mathrm{SL}-\mathrm{WSe}{ }_{2}$ estimated by the TF screening length; Derivation of Thomas Fermi Screening length in semiconductors; Transport properties of the SL$\mathrm{WSe}_{2}$ layer uncovered and covered with $\mathrm{C}_{60} \mathrm{~F}_{48}$; PL spectra of the $\mathrm{SL}-\mathrm{WSe}_{2}$ layer on graphite with and without $\mathrm{C}_{60} \mathrm{~F}_{48}$. This material is available free of charge via the Internet at http://pubs.acs.org.

\section{Competing financial interests}

The authors declare no competing financial interests.

\section{References:}

1. Bhimanapati, G. R.; Lin, Z.; Meunier, V.; Jung, Y.; Cha, J.; Das, S.; Xiao, D.; Son, Y.; Strano, M. S.; Cooper, V. R.; Liang, L.; Louie, S. G.; Ringe, E.; Zhou, W.; Kim, S. S.; Naik, R. R.; Sumpter, B. G.; Terrones, H.; Xia, F.; Wang, Y.; Zhu, J.; Akinwande, D.; Alem, N.; Schuller, J. A.; Schaak, R. E.; Terrones, M.; Robinson, J. A., Recent Advances in Two-Dimensional Materials beyond Graphene. ACS Nano 2015, 9, 11509-11539.

2. Liu, F.; Chow, W. L.; He, X.; Hu, P.; Zheng, S.; Wang, X.; Zhou, J.; Fu, Q.; Fu, W.; Yu, P.; Zeng, Q.; Fan, H. J.; Tay, B. K.; Kloc, C.; Liu, Z., Van der Waals p-n Junction Based on an Organic-Inorganic Heterostructure. Adv. Funct. Mater. 2015, 25, 5865-5871.

3. Duan, X.; Wang, C.; Fan, Z.; Hao, G.; Kou, L.; Halim, U.; Li, H.; Wu, X.; Wang, Y.; Jiang, J.; Pan, A.; Huang, Y.; Yu, R.; Duan, X., Synthesis of WS2xSe2-2x Alloy Nanosheets with CompositionTunable Electronic Properties. Nano Lett. 2016, 16, 264-269.

4. $\quad$ Alidoust, N.; Bian, G.; Xu, S. Y.; Sankar, R.; Neupane, M.; Liu, C.; Belopolski, I.; Qu, D. X.; Denlinger, J. D.; Chou, F. C.; Hasan, M. Z., Observation of Monolayer Valence Band Spin-Orbit Effect and Induced Quantum Well States in MoX2. Nat. Commun. 2014, 5, 4673.

5. Liu, Y.; Cai, Y.; Zhang, G.; Zhang, Y.-W.; Ang, K.-W., Al-Doped Black Phosphorus p-n Homojunction Diode for High Performance Photovoltaic. Adv. Funct. Mater. 2017, 27, 1604638.

6. Huang, Y. L.; Chen, Y.; Zhang, W.; Quek, S. Y.; Chen, C. H.; Li, L. J.; Hsu, W. T.; Chang, W. H.; Zheng, Y. J.; Chen, W.; Wee, A. T., Bandgap Tunability at Single-Layer Molybdenum Disulphide Grain Boundaries. Nat. Commun. 2015, 6, 6298.

7. Lu, C. P.; Li, G.; Mao, J.; Wang, L. M.; Andrei, E. Y., Bandgap, Mid-Gap States, and Gating Effects in MoS2. Nano Lett. 2014, 14, 4628-4633.

8. Huang, H.; Huang, Y.; Wang, S.; Zhu, M.; Xie, H.; Zhang, L.; Zheng, X.; Xie, Q.; Niu, D.; Gao, Y., Van Der Waals Heterostructures between Small Organic Molecules and Layered Substrates. Crystals 2016, 6, 113.

9. Jeong, H.; Oh, H. M.; Bang, S.; Jeong, H. J.; An, S. J.; Han, G. H.; Kim, H.; Yun, S. J.; Kim, K. K.; Park, J. C.; Lee, Y. H.; Lerondel, G.; Jeong, M. S., Metal-Insulator-Semiconductor Diode Consisting of Two-Dimensional Nanomaterials. Nano Lett. 2016, 16, 1858-1862.

10. Wan, W.; Li, X.; Li, X.; Xu, B.; Zhan, L.; Zhao, Z.; Zhang, P.; Wu, S. Q.; Zhu, Z.-z.; Huang, H.; Zhou, Y.; Cai, W., Interlayer Coupling of a Direct van der Waals Epitaxial MoS2/Graphene Heterostructure. RSC Adv. 2016, 6, 323-330.

11. Wi, S.; Kim, H.; Chen, M.; Nam, H.; Guo, L. J.; Meyhofer, E.; Liang, X., Enhancement of Photovoltaic Response in Multilayer MoS2 Induced by Plasma Doping. ACS Nano 2014, 8, 5270-5281. 
12. Withers, F.; Del Pozo-Zamudio, O.; Mishchenko, A.; Rooney, A. P.; Gholinia, A.; Watanabe, K.; Taniguchi, T.; Haigh, S. J.; Geim, A. K.; Tartakovskii, A. I.; Novoselov, K. S., Light-Emitting Diodes by Band-Structure Engineering in van der Waals Heterostructures. Nat. Mater. 2015, 14, 301-306.

13. Lin, J. D.; Han, C.; Wang, F.; Wang, R.; Xiang, D.; Qin, S.; Zhang, X.-A.; Wang, L.; Zhang, H.; Wee, A. T. S.; Chen, W., Electron-Doping-Enhanced Trion Formation in Monolayer Molybdenum Disulfide Functionalized with Cesium Carbonate. ACS Nano 2014, 8, 5323-5329.

14. Peimyoo, N.; Yang, W.; Shang, J.; Shen, X.; Wang, Y.; Yu, T., Chemically Driven Tunable Light Emission of Charged and Neutral Excitons in Monolayer WS2. ACS Nano 2014, 8, 11320-11329.

15. Tongay, S.; Zhou, J.; Ataca, C.; Liu, J.; Kang, J. S.; Matthews, T. S.; You, L.; Li, J.; Grossman, J. C.; Wu, J., Broad-Range Modulation of Light Emission in Two-Dimensional Semiconductors by Molecular Physisorption Gating. Nano Lett. 2013, 13, 2831-2836.

16. Yu, J.; Lee, C. H.; Bouilly, D.; Han, M.; Kim, P.; Steigerwald, M. L.; Roy, X.; Nuckolls, C., Patterning Superatom Dopants on Transition Metal Dichalcogenides. Nano Lett. 2016, 16, 3385-3389.

17. Marcia, M.; Hirsch, A.; Hauke, F., Perylene-Based Non-Covalent Functionalization of 2D Materials. FlatChem 2017, 1, 89-103.

18. Christodoulou, C.; Giannakopoulos, A.; Ligorio, G.; Oehzelt, M.; Timpel, M.; Niederhausen, J.; Pasquali, L.; Giglia, A.; Parvez, K.; Mullen, K.; Beljonne, D.; Koch, N.; Nardi, M. V., Tuning the Electronic Structure of Graphene by Molecular Dopants: Impact of the Substrate. ACS Appl. Mater. Interfaces 2015, 7, 19134-19144.

19. Huttmann, F.; Martinez-Galera, A. J.; Caciuc, V.; Atodiresei, N.; Schumacher, S.; Standop, S.; Hamada, I.; Wehling, T. O.; Blugel, S.; Michely, T., Tuning the van der Waals Interaction of Graphene with Molecules via Doping. Phys. Rev. Lett. 2015, 115, 236101.

20. Coletti, C.; Riedl, C.; Lee, D. S.; Krauss, B.; Patthey, L.; von Klitzing, K.; Smet, J. H.; Starke, U., Charge Neutrality and Band-gap Tuning of Epitaxial Graphene on SiC by Molecular Doping. Phys. Rev. $B$ 2010, 81, 235401.

21. Grüneis, A.; Vyalikh, D. V., Tunable Hybridization Between Electronic States of Graphene and a Metal Surface. Phys. Rev. B 2008, 77, 193401.

22. Amsalem, P.; Niederhausen, J.; Frisch, J.; Wilke, A.; Bröker, B.; Vollmer, A.; Rieger, R.; Müllen, K.; Rabe, J. r. P.; Koch, N., Metal-to-Acceptor Charge Transfer through a Molecular Spacer Layer. J. Phys. Chem. C 2011, 115, 17503-17507.

23. Wang, H.; Amsalem, P.; Heimel, G.; Salzmann, I.; Koch, N.; Oehzelt, M., Band-Bending in Organic Semiconductors: The Role of Alkali-Halide Interlayers. Adv. Mater. 2014, 26, 925-930.

24. Zheng, Y. J.; Huang, Y. L.; Chen, Y.; Zhao, W.; Eda, G.; Spataru, C. D.; Zhang, W.; Chang, Y.H.; Li, L.-J.; Chi, D., Heterointerface Screening Effects Between Organic Monolayers and Monolayer Transition Metal Dichalcogenides. ACS Nano 2016, 10, 2476-2484.

25. Jariwala, D.; Howell, S. L.; Chen, K. S.; Kang, J.; Sangwan, V. K.; Filippone, S. A.; Turrisi, R.; Marks, T. J.; Lauhon, L. J.; Hersam, M. C., Hybrid, Gate-Tunable, van der Waals p-n Heterojunctions from Pentacene and MoS2. Nano Lett. 2016, 16, 497-503.

26. Lee, W. H.; Park, J.; Sim, S. H.; Lim, S.; Kim, K. S.; Hong, B. H.; Cho, K., Surface-Directed Molecular Assembly of Pentacene on Monolayer Graphene for High-Performance Organic Transistors. $J$. Am. Chem. Soc. 2011, 133, 4447-4454.

27. Hill, H. M.; Rigosi, A. F.; Rim, K. T.; Flynn, G. W.; Heinz, T. F., Band Alignment in MoS2/WS2 Transition Metal Dichalcogenide Heterostructures Probed by Scanning Tunneling Microscopy and Spectroscopy. Nano Lett. 2016, 16, 4831-4837.

28. Chiu, M. H.; Zhang, C.; Shiu, H. W.; Chuu, C. P.; Chen, C. H.; Chang, C. Y.; Chen, C. H.; Chou, M. Y.; Shih, C. K.; Li, L. J., Determination of Band Alignment in the Single-Layer MoS2/WSe2 Heterojunction. Nat. Commun. 2015, 6, 7666.

29. Latini, S.; Winther, K. T.; Olsen, T.; Thygesen, K. S., Interlayer Excitons and Band Alignment in MoS2/hBN/WSe2 van der Waals Heterostructures. Nano Lett. 2017, 17, 938-945. 
30. Li, M.-Y.; Shi, Y.; Cheng, C.-C.; Lu, L.-S.; Lin, Y.-C.; Tang, H.-L.; Tsai, M.-L.; Chu, C.-W.; Wei, K.-H.; He, J.-H.; Chang, W.-H.; Suenaga, K.; Li, L.-J., Epitaxial Growth of a Monolayer WSe2MoS2 Lateral p-n Junction with an Atomically Sharp Interface. Science 2015, 349, 524-528.

31. Gong, Y.; Lei, S.; Ye, G.; Li, B.; He, Y.; Keyshar, K.; Zhang, X.; Wang, Q.; Lou, J.; Liu, Z.; Vajtai, R.; Zhou, W.; Ajayan, P. M., Two-Step Growth of Two-Dimensional WSe2/MoSe2 Heterostructures. Nano Lett. 2015, 15, 6135-6141.

32. Huang, C.; Wu, S.; Sanchez, A. M.; Peters, J. J.; Beanland, R.; Ross, J. S.; Rivera, P.; Yao, W.; Cobden, D. H.; Xu, X., Lateral Heterojunctions within Monolayer MoSe2-WSe2 Semiconductors. Nat. Mater. 2014, 13, 1096-1101.

33. Chen, K.; Wan, X.; Xu, J., Epitaxial Stitching and Stacking Growth of Atomically Thin Transition-Metal Dichalcogenides (TMDCs) Heterojunctions. Adv. Funct. Mater. 2017, 27, 1603884.

34. Smets, Y.; Stark, C. B.; Lach, S.; Schmitt, F.; Wright, C. A.; Wanke, M.; Ley, L.; Ziegler, C.; Pakes, C. I., Charge-Induced Distortion and Stabilization of Surface Transfer Doped Porphyrin Films. $J$. Chem. Phys. 2013, 139, 044703.

35. Huang, J.-K.; Pu, J.; Hsu, C.-L.; Chiu, M.-H.; Juang, Z.-Y.; Chang, Y.-H.; Chang, W.-H.; Iwasa, Y.; Takenobu, T.; Li, L.-J., Large-Area Synthesis of Highly Crystalline WSe2 Monolayers and Device Applications. ACS Nano 2014, 8, 923-930.

36. Huang, Y. L.; Ding, Z.; Zhang, W.; Chang, Y. H.; Shi, Y.; Li, L. J.; Song, Z.; Zheng, Y. J.; Chi, D.; Quek, S. Y.; Wee, A. T., Gap States at Low-Angle Grain Boundaries in Monolayer Tungsten Diselenide. Nano Lett. 2016, 16, 3682-3688.

37. Song, Z.; Wang, Q.; Lin, T.; Li, M. Y.; Li, L. J.; Zheng, Y. J.; Wang, Z.; Chi, D.; Ding, Z.; Huang, Y. L. Wee, A. T., Liquid-Solid Surface Phase Transformation of Fluorinated Fullerene on Monolayer Tungsten Diselenide, preparing.

38. Liu, Y.; Qiu, Z.; Carvalho, A.; Bao, Y.; Xu, H.; Tan, S. J.; Liu, W.; Castro Neto, A. H.; Loh, K. P.; Lu, J., Gate-Tunable Giant Stark Effect in Few-Layer Black Phosphorus. Nano Lett. 2017, 17, 19701977.

39. Kang, M.; Kim, B.; Ryu, S. H.; Jung, S. W.; Kim, J.; Moreschini, L.; Jozwiak, C.; Rotenberg, E.; Bostwick, A.; Kim, K. S., Universal Mechanism of Band-Gap Engineering in Transition-Metal Dichalcogenides. Nano Lett. 2017, 17, 1610-1615.

40. Zhang, Y.; Ugeda, M. M.; Jin, C.; Shi, S.-F.; Bradley, A. J.; Martín-Recio, A.; Ryu, H.; Kim, J.; Tang, S.; Kim, Y.; Zhou, B.; Hwang, C.; Chen, Y.; Wang, F.; Crommie, M. F.; Hussain, Z.; Shen, Z.-X.; Mo, S.-K., Electronic Structure, Surface Doping, and Optical Response in Epitaxial WSe2 Thin Films. Nano Lett. 2016, 16, 2485-2491.

41. Resta, R., Thomas-Fermi Dielectric Screening in Semiconductors. Phys. Rev. B 1977, 16, $2717-$ 2722.

42. Scharf, B.; Wang, Z.; Tuan, D. V.; Shan, J.; Mak, K. F.; Zutic, I.; Dery, H., Probing Many-Body Interactions in Monolayer Transition-Metal Dichalcogenides. //arxiv.org/abs/1606.07101 2016.

43. Vanderbilt, D., Soft Self-Consistent Pseudopotentials in a Generalized Eigenvalue Formalism. Phys. Rev. B 1990, 41, 7892-7895.

44. Blöchl, P. E., Projector Augmented-Wave Method. Phys. Rev. B 1994, 50, 17953-17979.

45. Perdew, J. P.; Burke, K.; Ernzerhof, M., Generalized Gradient Approximation Made Simple. Phys. Rev. Lett. 1996, 77, 3865.

46. Kresse, G.; Hafner, J., Ab Initio molecular Dynamics for Liquid Metals. Phys. Rev. B 1993, 47, 558-561.

47. Dion, M.; Rydberg, H.; Schroder, E.; Langreth, D. C.; Lundqvist, B. I., van der Waals Density Functional for General Geometries. Phys. Rev. Lett. 2004, 92, 246401.

48. Klimes, J.; Bowler, D. R.; Michaelides, A., Chemical Accuracy for the van der Waals Density Functional. J. Phys. Condens. Matter 2010, 22, 022201. 

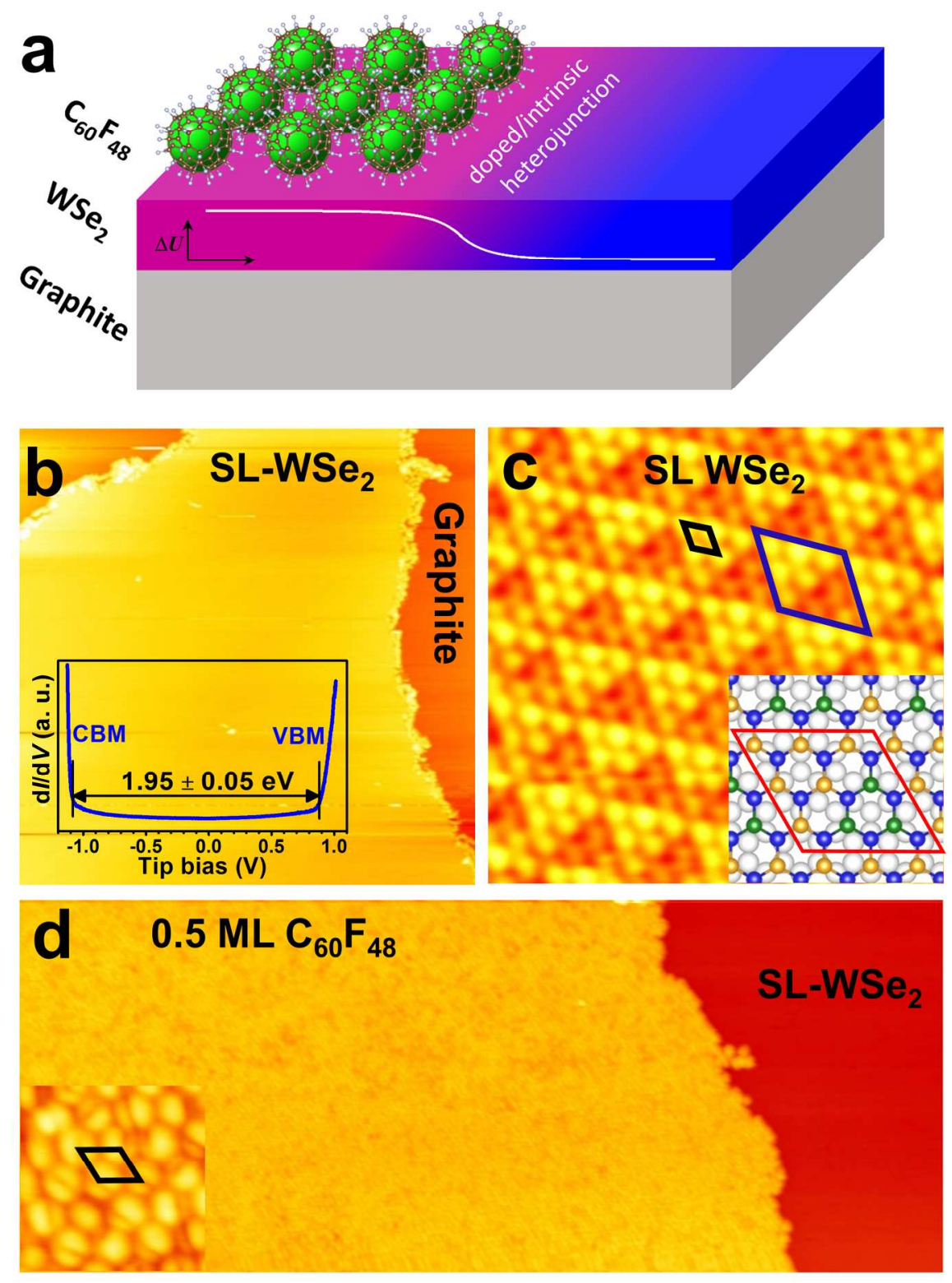

Figure 1. Fabrication of organic/TMD/graphite heterojunction. (a) A schematic model of the C60F48/SLWSe2/graphite heterostructure. (b) A large-scale STM image of a SL-WSe2 flake on graphite $(250 \times 250$ $\mathrm{nm} 2$; VTip $=-3.1 \mathrm{~V}$ ). Inset: STS spectrum reveals a $1.95 \mathrm{eV}$ bandgap for the clean SL-WSe2 (set point: VTip $=-0.9 \mathrm{~V}$, ITip $=100 \mathrm{pA})$. (c) Moiré superstructure is visible on the SL-WSe2 $(6 \times 6 \mathrm{~nm} 2 ; \mathrm{VTip}=-0.8 \mathrm{~V})$. The proposed model corresponds to the moiré superstructure is shown in the inset, where the Se atoms on the top/hollow sites of the graphite are represented by gold/green. Blue and grey atoms represent W and C respectively. (d) $0.5 \mathrm{ML}$ C60F48 molecules form close-packed islands on the SL-WSe2/graphite surface $(80 \times 200 \mathrm{~nm} 2 ; \mathrm{VTip}=-3.1 \mathrm{~V})$. The zoom-in of the close-packed island is shown in the bottom left inset in (d) $(6 \times 6 \mathrm{~nm} 2 ;$ VTip $=-3.1 \mathrm{~V})$. 

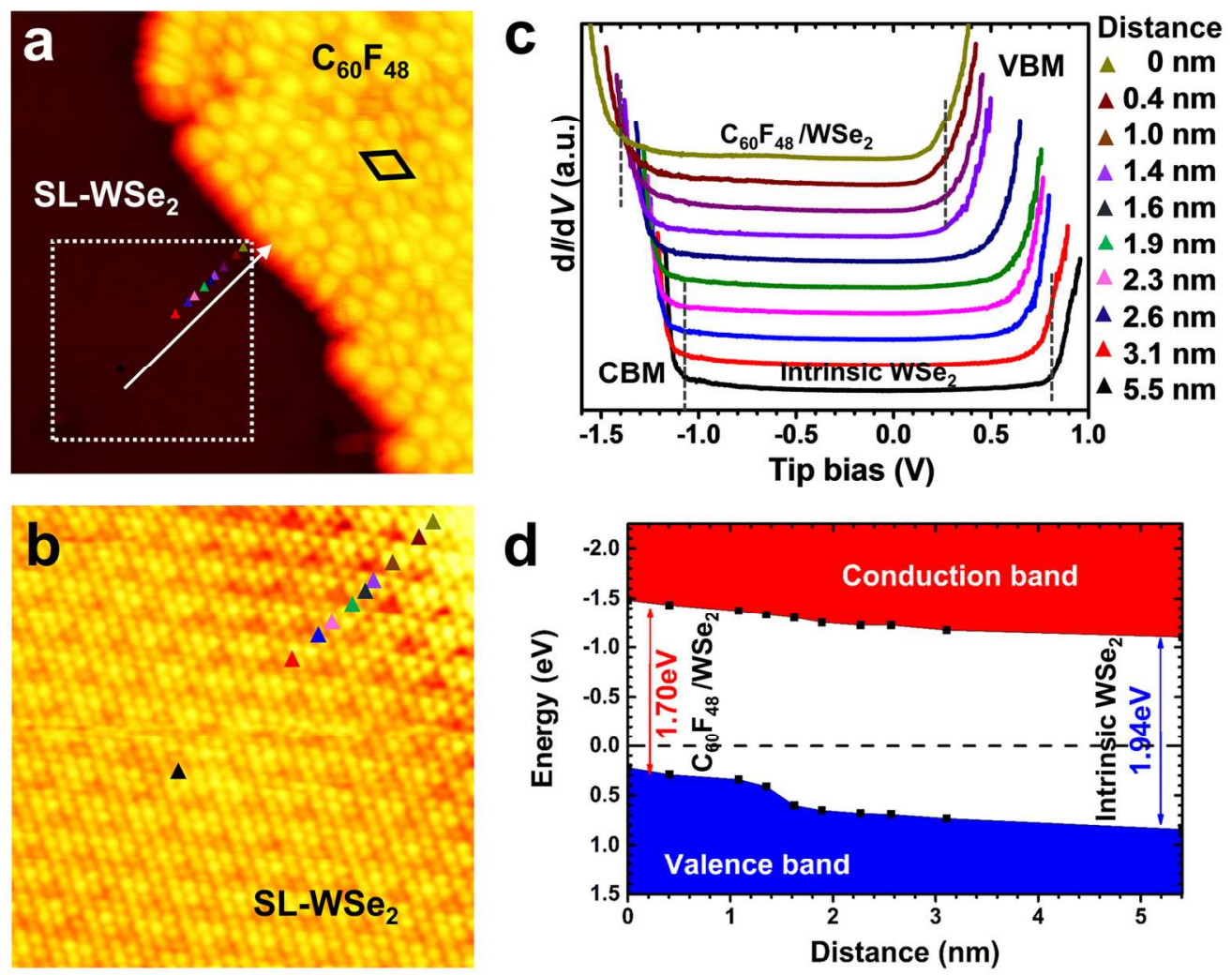

Figure 2. 1D intrinsic/p-doped heterojunction in SL-WSe2. (a) A molecular-resolved STM image shows a close-packed C60F48 island (0.5 ML) on the SL-WSe2 $(20 \times 20 \mathrm{~nm} 2$; VTip $=-3.1 \mathrm{~V})$, and (b) is the close-up corresponding to the white dotted square in the bare SL-WSe2. Contrast variation is observed close to the molecular island edge $(8 \times 8 \mathrm{~nm} 2$, VTip $=-0.9 \mathrm{~V})$. (c) dI/dV spectra recorded at the positions denoted by the multicolored triangles in panel (a) and (b) (set point: VTip $=-0.9 \mathrm{~V}$, ITip $=100 \mathrm{pA}$ ). The variation of the WSe2 bandgap is getting pronounced as the STM tip approaches to the C60F48 island. (d) Schematic diagram shows the band bending of the SL-WSe2 as a function of the distance from the molecular island, due to the TF screening effect. 
a

b $\quad \Delta Q(-e)$
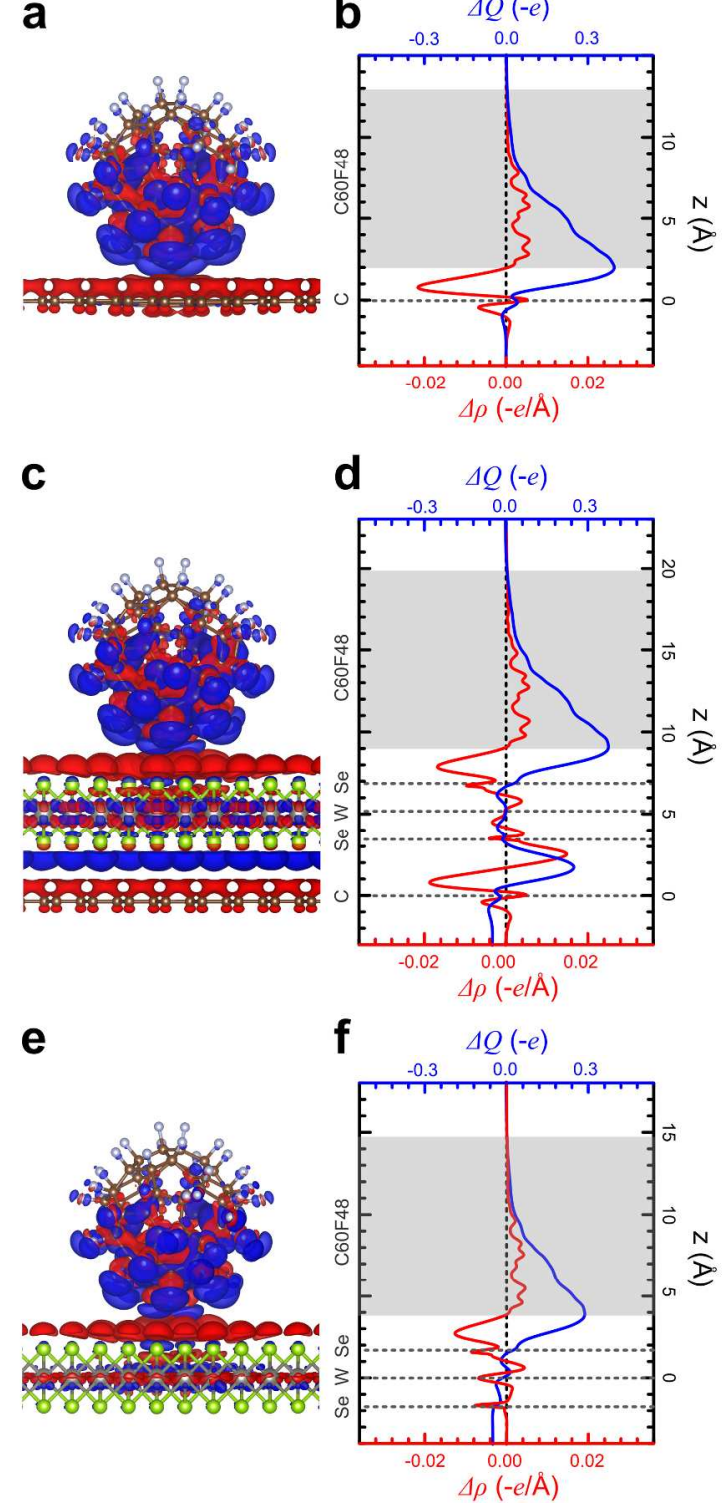

Figure 3. Calculations of the charge transfer. (a), (c) and (e) are side views of DCD isosurfaces of a C60F48 molecule on a single-layer graphene, SL-WSe2/graphene, and SL-WSe2, respectively (isovalue: \pm 0.001 e/ $\AA 3)$. A $(7 \times 7)$ graphene supercell and a $(3 \sqrt{ } 3 \times 3 \sqrt{ } 3)$ WSe2 supercell were used. Gray, green, brown and pearl gray balls represent $\mathrm{W}, \mathrm{Se}, \mathrm{C}$ and $\mathrm{F}$ atoms, respectively. Blue (red) represents electron accumulation (depletion) regions. (b), (d) and (f) represent the corresponding plots of the plane-averaged $\Delta \rho(z)$ (red) and $\Delta \mathrm{Q}(\mathrm{z})$ (blue). 


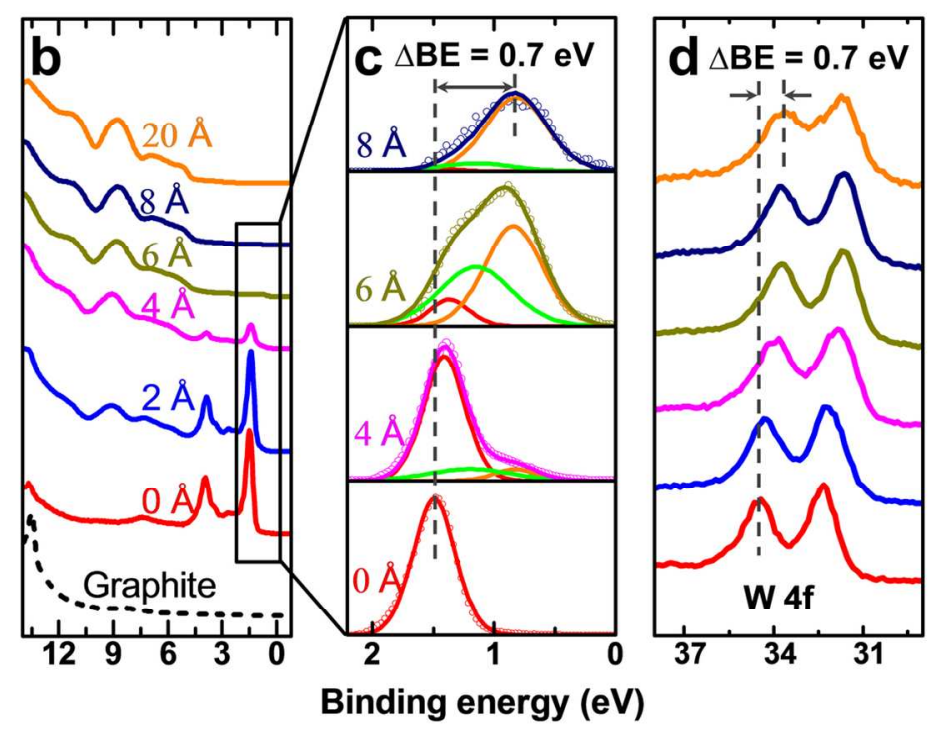

Figure 4. PES spectra of the C60F48/WSe2/graphite heterostructure. (a) The secondary cut-off shifts to higher kinetic energies with the increase of C60F48 coverage, indicating a WF increase. (b) Valence band spectra show that the sharp WSe2 peaks are pronounced at around $1.5 \mathrm{eV}$ and $4 \mathrm{eV}$ before molecular deposition $(0 \AA)$, and strongly attenuated for coverages higher than $4 \AA$. The dashed black line in (a) and (b) represents the clean graphite for comparison. (c) Zoom-in spectra of the valence region after Shirleybackground subtraction and normalization. Three different contributions with fixed energetic positions can be clearly separated. The red peak stems from the uncovered WSe2, the orange peak is shifted by $0.7 \mathrm{eV}$ to lower BE and stems from the WSe2 covered by C60F48, and the green peak stems from the transition region between covered and uncovered WSe2. (d) W $4 \mathrm{f}$ core levels shift by 0.7 eV to lower BE with C60F48 coverage increasing. 

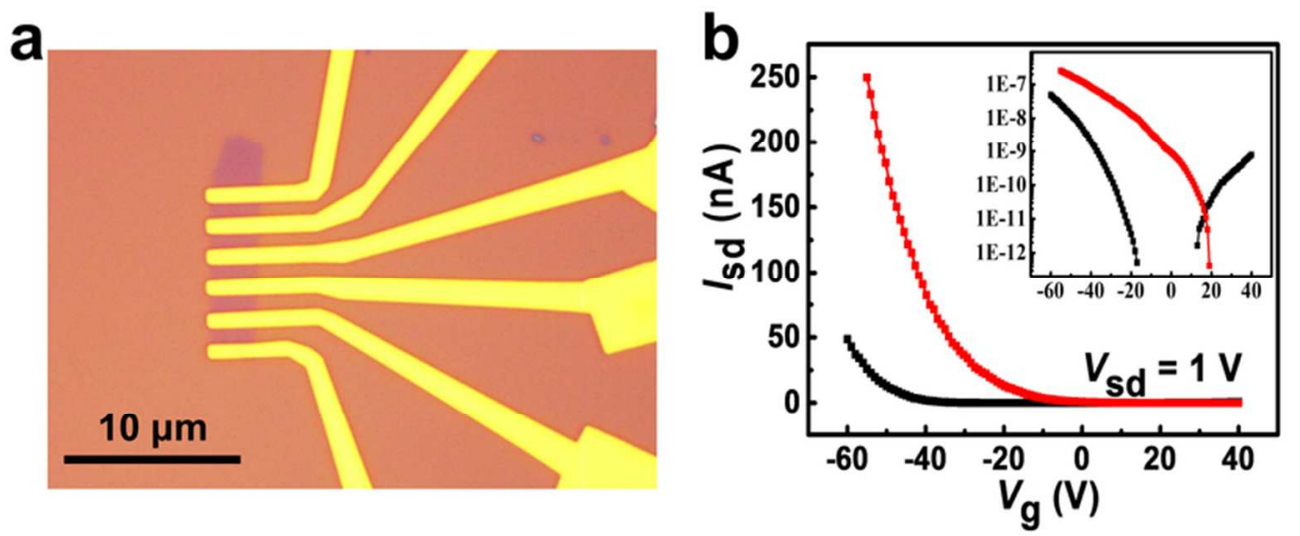

Figure 5. Transport measurements on a SL-WSe2 FET without and with C60F48. (a) Optical image of the asfabricated WSe2 device. (b) Transfer characteristics (Isd-Vg) of the SL-WSe2 device before (black) and after $20 \mathrm{~nm}$ C60F48 deposition (red) with Vsd = $1 \mathrm{~V}$. Inset: logarithmic plot of the transfer curve.

$70 \times 29 \mathrm{~mm}(300 \times 300 \mathrm{DPI})$ 


1
1
3
4
5
6
7
8
9
10
11
12
13
14
15
16
17
18
19
20
21
22
23
24
25
26
27
28
29
30
31
32
33
34
35
36
37
38
39
40
41
42
43
44
55
50
56
57
48
59
50
51
53
50

$61 \times 29 \mathrm{~mm}(300 \times 300$ DPI $)$ 